\title{
Proof of concept study to identify candidate biomarkers of fibrosis using high throughput peptide aptamer microarray and validate by enzyme linked immunosorbant assay
}

\author{
Sam Straw $^{1 *}$, Paul Ko Ferrigno ${ }^{2,3}$, Qifeng Song $^{2}$, Darren Tomlinson ${ }^{4}$, Francesco Del Galdo ${ }^{1}$ \\ ${ }^{1}$ Scleroderma Research Centre, Leeds Institute of Molecular medicine, St James's University Hospital, Leeds, UK \\ ${ }^{2}$ Section of Experimental Therapeutics, Leeds Institute of Molecular medicine, St James's University Hospital, Leeds, UK \\ ${ }^{3}$ Discovery Technology, Avacta Group plc, Wetherby, UK \\ ${ }^{4}$ Bioscreening Technology, Faculty of Biological Sciences, Institute of Molecular and Cellular Biology, University of Leeds, Leeds, \\ UK \\ Email: ${ }^{*}$ um08ss@leeds.ac.uk
}

Received 19 June 2013; revised 24 July 2013; accepted 4 August 2013

Copyright (C) 2013 Sam Straw et al. This is an open access article distributed under the Creative Commons Attribution License, which permits unrestricted use, distribution, and reproduction in any medium, provided the original work is properly cited.

\begin{abstract}
Background: Nine proteins were identified as putative profibrotic biomarkers in systemic sclerosis (SSc) and an unrelated fibrotic disease in a previously published proteomic study. As the majority of these proteins were orphans of commercially available antibodies, the nine proteins were investigated to determine whether binding peptide aptamers of the Stefin A quadruple mutant-Tracy variant (referred to as "affimers") could be validated by enzyme linked immunosorbant assay (ELISA) to allow the quantification of these candidate biomarkers in the sera of SSc patients. Materials and Methods: Candidate biomarker peptides were analysed by high throughput affimer microarray to identify binding affimers. Two candidate biomarkers were prioritised, and binding affimers were expressed from genetically modified BL21 competent $E$. coli strains and purified. These affimers were used in indirect ELISA, and then sandwich ELISA formats against the candidate biomarker recombinant proteins osteonectin and pigment epithetlium-derived factor (PEDF). Results: 39 affimers were identified as binders for eight of the nine candidate biomarker peptides were by affimer microarray; six for osteonectin and eleven for PEDF. Two of the six and all eleven were able to recognize physiological concentrations (5 and $1 \mu \mathrm{g} \cdot \mathrm{ml}^{-1}$ ) of osteonectin and PEDF, respectively by indirect ELISA. In sandwich ELISA format: two affimers were able to detect recombinant PEDF; however, the two affimers identi-
\end{abstract}

"Corresponding author. fied in indirect ELISA were unable to recognise recombinant osteonectin, and were thus hypothesised to bind to osteonectin at the same binding site. Discussion: SSc is currently an orphan of fully validated biomarkers, which is required for the development of stratified medicine in this field. This approach has laid the groundwork for an affimer based on multiplexed assay, to validate biomarkers in the sera of SSc patients in the future.

Keywords: Fibrosis; Biomarker; Peptide Aptamer; Systemic Sclerosis; Proof of Concept

\section{INTRODUCTION}

Fibrotic disorders, which include systemic sclerosis (SSc) and the newly recognized nephrogenic systemic fibrosis (NSF), are conditions resulting from the abnormal production and deposition of collagen and other extracellular matrix proteins by aberrantly activated fibroblasts [14]. SSc is an idiopathic, autoimmune disorder in which deposition occurs in the skin and internal organs, alongside a fibro proliferative vasculopathy $[1,2,5]$. NSF is in a non-autoimmune, non-vasculopathic condition resulting from exposure to gadolinium-containing contrast agents used in magnetic resonance imaging (MRI), in patients with reduced renal function $[3,6]$. These conditions are unrelated to many of their clinical features and underlying aetiology, but have a shared alteration to a profibrotic fibroblast phenotype [3].

Fully validated biomarkers, which assist early diagnosis, allow patient stratification and provide a predictive prognostic value, are not available for SSc $[2,7,8]$. The 
current gold standard prognostic test used in clinical trials, the modified Rodnan skin score (MRSS) is flawed in terms of accuracy and reproducibility between observers [7,9-11].

Del Galdo et al. [3] hypothesized that shared alterations in the proteome of cultured, explanted dermal fibroblasts taken from patients with SSc and NSF as opposed to healthy controls would reflect the proteins involved in the profibrotic fibroblast phenotype regardless of the triggering event, whether environmental exposure or autoimmune disease. The authors identified nine proteins: reticulocalbin-3, osteonectin, $\alpha 2$ chain of type I collagen, reticulocalbin-1, tropomyosin 4 , enolase 1 , calreticulin precursor, actin $\alpha 1$, pigment epithelium derived factor (PEDF), which if validated, may act as putative fibroblast profibrotic activity biomarkers [3].

Traditionally, protein biomarkers have been validated by the use of antibodies [12]. Although antibodies are highly specific and have high affinity for their targets, these molecules have a number of limitations:

- Antibodies are large, complex molecules which require multiple disulfide bond formation and posttranslational glycosylation to attain stability and are thus difficult to express and scale up in vitro [13-15].

- The large size of antibodies may lead to steric hindrance and limit their utility in certain applications [13].

- The constant region of the antibody facilitates many intracellular interactions and therefore leads to high levels of 'background' signal in multiplexed assays [13].

- Antibody molecules have evolved to function in solution, whereas most clinical assays are performed upon a surface [13].

- Antibodies are raised in animal hosts, which imply several ethical considerations, namely whether it is expensive and limited practically as not all proteins are immunogenic due to evolutionary conservation between humans and the host [13].

- Antibodies are only commercially available for a very small percentage of the entire proteome [13] and the generation of novel antibodies is relatively slow, so is a rate limiting step in the validation of candidate biomarkers [16].

Peptide aptamers are artificial peptide recognition moieties, which are analogous to antibodies. They are proteins with inserted random short peptide sequences, which are expressed as parts of the primary sequence of a structurally stable protein scaffold and act as ligands to bind target proteins [16], created by the insertion of random oligonucleotides into a restriction site in the open reading frame of the scaffold in an organism engineered to express them [14]. One group has developed various scaffold variants based on stefin A (cystatin A), a cathep- sin protease inhibitor. Their latest variant: stefin A quadruple mutant-Tracy (SQT) [15] has several important properties:

- It is biologically neutral, as the restriction site was chosen so as to express the inserted peptide in what would otherwise be a protein-protein interaction site, thus losing the protease inhibitor activity [17].

- It accepts peptide insertions at three points, which are distant in the primary structure but adjacent in the tertiary structure of the protein, each of which may interact with binding molecules differently, thus increasing the specificity of the peptide aptamers to their targets $[15,17]$.

- It is unusually stable on surfaces and so may be printed into microarray format a lot easier than antibodies [13].

- They display exquisite specificity and binding affinities comparable to antibodies $\mathrm{K}_{\mathrm{d}} 10^{-6}-5 \times 10^{-9} \mathrm{M}$ compared to $\mathrm{K}_{\mathrm{d}} 10^{-7}-10^{-11} \mathrm{M}$. As due to the constrained nature of the binding peptide, the conformational entropy lost upon binding is very low $[12,13]$.

- It has been developed with a hexa-histidine tag allowing purification following expression [13].

Here we use the term "affimers" for peptide aptamers derived from the SQT variant $[15,18]$. Song et al. have developed a BL21 competent E. coli expression library with 17,000 strains, genetically modified to synthesise a random affimer and resistant to the antibiotics kanamycin and chlroamphenicol [19]. Alongside this, the authors developed a microarray with corresponding random affimers printed in duplicate ontonickel-nitrilotriacetic acid (Ni-NTA) coated glass slides (Xenopore) [13]. This microarray and similar proof of concept microarrays have previously been shown to allow the detection of certain proteins in patient samples [13]. The genetic sequence of $E$. coli strains, and therefore amino acid sequence of random peptide aptamers is unknown until characterisation, and so random affimers may be referred as "naïve affimers".

Here we aimed to determine whether:

1) That binding affimers to the nine candidate biomarker proteins identified by Del Galdo et al. [3] may be identified by high throughput affimer microarray.

2) An enzyme-linked immunosorbant assay (ELISA) protocol may be optimized using affimers identified from microarray in place of antibodies for the detection of candidate biomarkers proteins.

3) Affimers may be identified which detect the protein biomarkers in sandwich ELISA format without the use of antibodies, thus allowing the development of multiplexed affimer based on biomarker detection in the future, i.e. detection of multiple biomarkers in a clinical sample.

\section{MATERIALS AND METHODS}




\subsection{Probingof Naïve Affimer Microarray with Candidate Biomarker Peptides}

The affimer microarray was first blocked by incubation with $3 \%$ bovine serum albumin (BSA) in phosphate buffered salinesolution (PBS) with $0.1 \%$ [v/v] Tween 20 (PBST) at room temperature with shaking on reciprocal mixer at $60 \mathrm{rpm}$ for $1 \mathrm{~h} 45 \mathrm{~min}$. Following blocking, the microarray slides were incubated separately with nine candidate biomarker peptides (see introduction) which had been synthesized and biotinylated by a third party (China Peptides) at a concentration of $300 \mathrm{ng} \cdot \mathrm{ml}^{-1}$ in 2 $\mathrm{ml}$ PBST. Incubation was performed at room temperature, on reciprocal mixer at $60 \mathrm{rpm}$ for $1 \mathrm{~h}$. Control microarray was carried out without biotin linked candidate biomarker peptide to detect affimers, which bound to strepta-vidincy 3 conjugate (see below).

One peptide, PEDF, did not generate "hits"and so recombinant human PEDF (Abcam) was biotinylated by amine coupling and then probed as above. Briefly, $1 \mu \mathrm{g}$ PEDF was dissolved in $50 \mu \mathrm{l}$ PBS and $0.8 \mu \mathrm{l}$ EZ-link NHS-SS-biotin (Pierce) was added at a concentration of $0.2 \mathrm{mg} \cdot \mathrm{ml}^{-1}$ in dimethylsulfoxide solution (DMSO) and incubated for $1 \mathrm{~h}$. A bio-spin 6 chromatography column (Bio-Rad) was centrifuged at $1000 \mathrm{~g}$ for $2 \mathrm{~min}$, sample added and then spun again at $1000 \mathrm{~g}$ for $4 \mathrm{~min}$ to desalt biotin from the PEDF sample as per the manufacturer's instructions [20]. Biotinylation was confirmed by ELISA. Briefly, samples were added alongside a known concentration of PEDF in PBS. Mouse anti-PEDF (Merck) was incubated at a concentration of 1 in 1000 in 3\% BSA in PBS and then sheep anti-mouse horseradish peroxidase (HRP) conjugate (Sigma) to detect the presence of PEDF, and separately with streptavidin-HRP (BD Biosciences) 1 in 1000 in 3\% BSA in PBS to detect the presence of biotin. All reagents were incubated for $1 \mathrm{~h}$ at room temperature, with washing with PBST [v/v] 0.05\% four times between incubations. $50 \mu \mathrm{l}$ 3,3',5,5'-tertramethl-benzidine (TMB) was added and reaction arrested with $50 \mu \mathrm{l}$ $0.5 \mathrm{M}$ hydrochloric acid $(\mathrm{HCl})$ after observable colour change and optical density measured at $450 \mathrm{~nm}$ (OD450) by Multiskan EX plate reader and Ascent software.

After washing three times with PBST at room temperature on reciprocal mixer at $60 \mathrm{rpm}$ for $5 \mathrm{~min}$, microarray slides were incubated with streptavidin-cy3 conjugate (Invitrogen) (1 in 4000, $2 \mathrm{ml} \mathrm{3 \%} \mathrm{BSA} \mathrm{in} \mathrm{PBST)}$ at room temperature on reciprocal mixer at $60 \mathrm{rpm}$ for 1 $\mathrm{h}$ (whilst avoiding exposure to light). Microarray slides were then washed as above, three times with PBST and three times with distilled water to remove sodium chloride $(\mathrm{NaCl})$ from the microarray. Water was removed by centrifugation at $4^{\circ} \mathrm{C}, 1800 \mathrm{rpm}, 2 \mathrm{~min}$ (Eppendorf 5810 $\mathrm{R}$ centrifuge). A standard DNA microarray scanner (Axon) and Genepix 4.0 software was used to quantify the signal intensity for each spot, using the settings: PMT gain 400, power $33 \%$, pixel size 10 in accordance with the previously developed protocol [13]. Only spots with the following criteria were considered to represent a naïve affimers with binding affinity for a particular candidate biomarker peptide, henceforth referred to as "hits":

- Signal (foreground) $>$ [signal (background) +2 standard deviations signal (background)].

- 2 adjacent spots, as affimers were printed in duplicate.

- Specific for a particular candidate biomarker. Many spots were non-specific, having affinity for more than one biotin linked candidate biomarker or streptavidin (control).

\subsection{E. coli Expression of Naïve Affimers}

Due to commercially available antibodies to the proteins osteonectin and PEDF, these proteins were prioritized for further investigation until a non-antibody validation protocol could be optimized.

Expression and purification of naïve affimers was conducted by the method developed by Song et al. [13] with some modifications. Hits obtained were matched with corresponding BL21 competent $E$. coli strains in the affimer expression library developed by Song et al. [19] and a starter cell culture was madewith $5 \mathrm{ml}$ Luria-Beltani (LB) medium (10 $\mathrm{g} \cdot \mathrm{l}^{-1}$ triyptone, $10 \mathrm{~g} \cdot \mathrm{l}^{-1} \mathrm{NaCl}, 5$ $\mathrm{g} \cdot \mathrm{l}^{-1}$ yeast extract in distilled water, autoclaved at $121^{\circ} \mathrm{C}$ for $20 \mathrm{~min}$ ) supplemented with $30 \mu \mathrm{g} \cdot \mathrm{ml}^{-1}$ chloramphenicol and $30 \mu \mathrm{g} \cdot \mathrm{ml}^{-1}$ kanamycin. Cells were allowed to grow overnight in a Brunswick mixer at $37^{\circ} \mathrm{C}$ and 250 rpm. Cell cultures were then expanded 100-fold in 500 $\mathrm{ml} \mathrm{LB}$ medium with supplemented $30 \mu \mathrm{g} \cdot \mathrm{ml}^{-1}$ chloramphenicol and $30 \mu \mathrm{g} \cdot \mathrm{ml}^{-1}$ kanamycin and grown to OD600 0.2 - 0.3 measured by spectrophotometer. To induce the expression of affimers, isopropyl- $\beta$-D-thioglactoside (IPTG) was added at a concentration of $0.1 \mathrm{mM}$ and induced at $37^{\circ} \mathrm{C}$ and $250 \mathrm{rpm}$ for 2 h. E. coli were harvested from culture by centrifugation at $4000 \mathrm{rpm}$ at $4^{\circ} \mathrm{C}$ for $5 \mathrm{~min}$. Supernatant was discarded and pellets were stored at $-20^{\circ} \mathrm{C}$ or immediately purified.

\subsection{Affimer Purification}

Harvested E. coli were suspended in $20 \mathrm{ml}$ lysis buffer $(0.1 \mathrm{M}$ sodium phosphate solution, $\mathrm{pH} 7.4$ with $300 \mathrm{mM}$ $\mathrm{NaCl}, 1$ in 10 pop culture (Novagen) and benzonase (Novagen) at 5 unitsml-1) and incubated at room temperature on reciprocal mixer at $60 \mathrm{rpm}$ for $30 \mathrm{~min}$. Cell debris was removed by centrifugation at $4000 \mathrm{rpm}, 4^{\circ} \mathrm{C}$ for $20 \mathrm{~min} .1 \mathrm{ml}$ his-select cobalt affinity gel (Sigma) was added to a 45 - $90 \mu \mathrm{m}$ chromatography column (Evergreen Scientific) and washed twice with $10 \mathrm{ml} 0.1 \mathrm{M}$ sodium phosphate $\mathrm{pH} 7.4$ with $300 \mathrm{mM} \mathrm{NaCl}$ to remove ethanol. Supernatant was added to the chromatography 
column and mixed with his-select cobalt affinity gel for 1 $\mathrm{h}$ at $4^{\circ} \mathrm{C}$ to allow binding of affimers (see introduction). Non-specific binding was removed by washing ten times with $10 \mathrm{ml} 0.1 \mathrm{M}$ sodium phosphate $\mathrm{pH} 7.4$ with 300 $\mathrm{mM} \mathrm{NaCl}$ with agitation by pipetting every five washes.

Affimers bound to his-tag cobalt gel were eluted by adding $800 \mu$ l elution buffer (PBS with $300 \mathrm{mM} \mathrm{NaCl}$, $0.25 \mathrm{M}$ imidazole), mixing briefly and incubating for 3 min before elution. The concentration of affimers and contaminant E. coli proteins was determined with measurement by Nanodrop (setting: protein A280), and elution repeated until the concentration fell below $0.1 \mathrm{mg} \cdot \mathrm{ml}^{-1}$.

The purity of obtained affimers was determined by polyacrylamide gel electrophoresis with $15 \%$ sodium dodecyl sulphate, followed by staining with coomassie stain (Thermo Scientific) for $3-4 \mathrm{~h}$ at room temperature or $4{ }^{\circ} \mathrm{C}$ overnight. Following this, destaining with $20 \%$ $[\mathrm{v} / \mathrm{v}]$ methanol and $10 \%[\mathrm{v} / \mathrm{v}]$ acetic acid in distilled water, and then equilibrilation in a storage solution of $5 \%$ [v/v] acetic acidin distilled water for $1 \mathrm{~h}$. Samples were qualitatively assessed for the presence of one band at 11 $\mathrm{kDa}$ with minimal contaminant $E$. coli proteins. Expression and purification of affimers was repeated for samples judged to be qualitatively impure.

\subsection{Indirect Affimer ELISA Validation Protocol Optimisation}

The use of affimers in ELISA format is a novel approach. Previously, naïve affimers identified from microarray have been compiled into a smaller microarray format and used to probe for their targets in patient sample [13], however in the future multiplexed assays will be performed in ELISA format, thus ELISA protocol optimization was essential.

The applicability of the affimers also depends upon their ability to bind to the (folded) recombinant protein, as well as the (linear) peptide, and so recombinant proteins were used in the development of the ELISA protocol. Thus the bulk of the project was to develop a protocol for the validation of binding affimers by indirect and then sandwich ELISA.

The indirect ELISA protocol was developed with the recombinant osteonectin protein and appropriate binding affimers identified from microarray (see Table 1). Briefly, initial approaches involved utilising the surface binding properties of affimers by applying these to a high binding 96-well plate (Costar) in decreasing concentrations $\left(0.4,0.2,0.1,0.05\right.$ and $\left.0 \mathrm{mg} \cdot \mathrm{ml}^{-1}\right)$. This was followed by incubation with a consistent concentration of recombinant osteonectin $\left(5 \mu \mathrm{g} \cdot \mathrm{ml}^{-1}\right.$ in PBS). This produced unremarkable results and so the experiment was repeated, with a consistent concentration of affimer $(0.2$ $\mathrm{mg} \cdot \mathrm{ml}^{-1}$ ) but with a decreasing concentration of recombinant osteonectin $\left(5,2.5,1.25,0.63,0.31,0 \mu \mathrm{g} \cdot \mathrm{ml}^{-1}\right)$, again producing unremarkable results. Repetition of the
Table 1. Identification of affimers for osteonectin and PEDF which were further investigated. Affimer identifications correspond to hits obtained from microarray (data not shown). Identification code gives the location of affimer within the E. coli expression library, for example: P1A1 = plate 1 , row A, column 1 .

\begin{tabular}{ll}
\hline Candidate biomarker & Identification of affimer \\
\hline \multirow{3}{*}{ Osteonectin } & P17A7 \\
& P17M5 \\
& P17D8 \\
& P17P6 \\
& P9I2 \\
& P3E1 \\
& P3K13 \\
& P3K19 \\
& P3F15 \\
& P3I23 \\
P4K16 \\
Pigment epithelial derived & P5N16 \\
factor (PEDF) & P14O4 \\
& P14J4 \\
& P17I18 \\
& P11P19 \\
\hline
\end{tabular}

previous experiment with a greater concentration of affimer $\left(0.8 \mathrm{mg} \cdot \mathrm{ml}^{-1}\right)$ failed, as did repetition with denatured osteonectin, hypothesized to present affimer binding sites hidden within the folded protein. Coating the surface of the 96-well plate with recombinant osteonectin was the next approach utilized. However, the initial experiment failed, most probably due to the presence of BSA in the initial coating solution, which outcompeted the recombinant osteonectin to bind to the wells.

The final approach utilized osteonectin dissolved in PBS to coat the wells and over several attempts the experimental conditions were modified including changes to: reagents, incubation times, temperatures, washing reagents and repetitions. The final protocol is detailed below.

\subsection{Indirect Affimer ELISA}

A Cartoon summarizing this procedure is shown in Figure 1. A high binding 96-well plate (Costar) was incubated with $50 \mu \mathrm{l}$ recombinant osteonectin in PBS at concentrations of 5,1 , and $0 \mu \mathrm{g} \cdot \mathrm{ml}^{-1}$ overnight at $4^{\circ} \mathrm{C}$. Recombinant osteonectin was discarded and the wells were blocked by applying $100 \mu 13 \%$ BSA in PBS and incubating for $3 \mathrm{~h}$ at $37^{\circ} \mathrm{C}$. Wells were washed by discarding the solution, then applying and discarding $100 \mu \mathrm{PBST}$ [v/v] $0.05 \%$ four times. A consistent concentration of each affimer was added at $0.2 \mathrm{mg} \cdot \mathrm{ml}^{-1}$, and incubated for $1 \mathrm{~h}$ at room temperature, with mixing on ELISA plate mixer at $250 \mathrm{rpm}$. The plate was washed as above and incubated with $50 \mu \mathrm{l}$ rabbit anti-cystatin A 1 in 1000 in $3 \%$ BSA in PBS (anti-cystain A is an antibody against the SQT scaffold) for $1 \mathrm{~h}$ as above. The plate was washed and then incubated with $50 \mu \mathrm{l}$ anti-rabbit horse HRP conjugate 1 in 5000 in 3\% BSA in PBS. After a final washing step, $50 \mu \mathrm{l}$ TMB was added to each well. The reaction was arrested by $50 \mu \mathrm{l} 0.5 \mathrm{M} \mathrm{HCl}$ after no- 


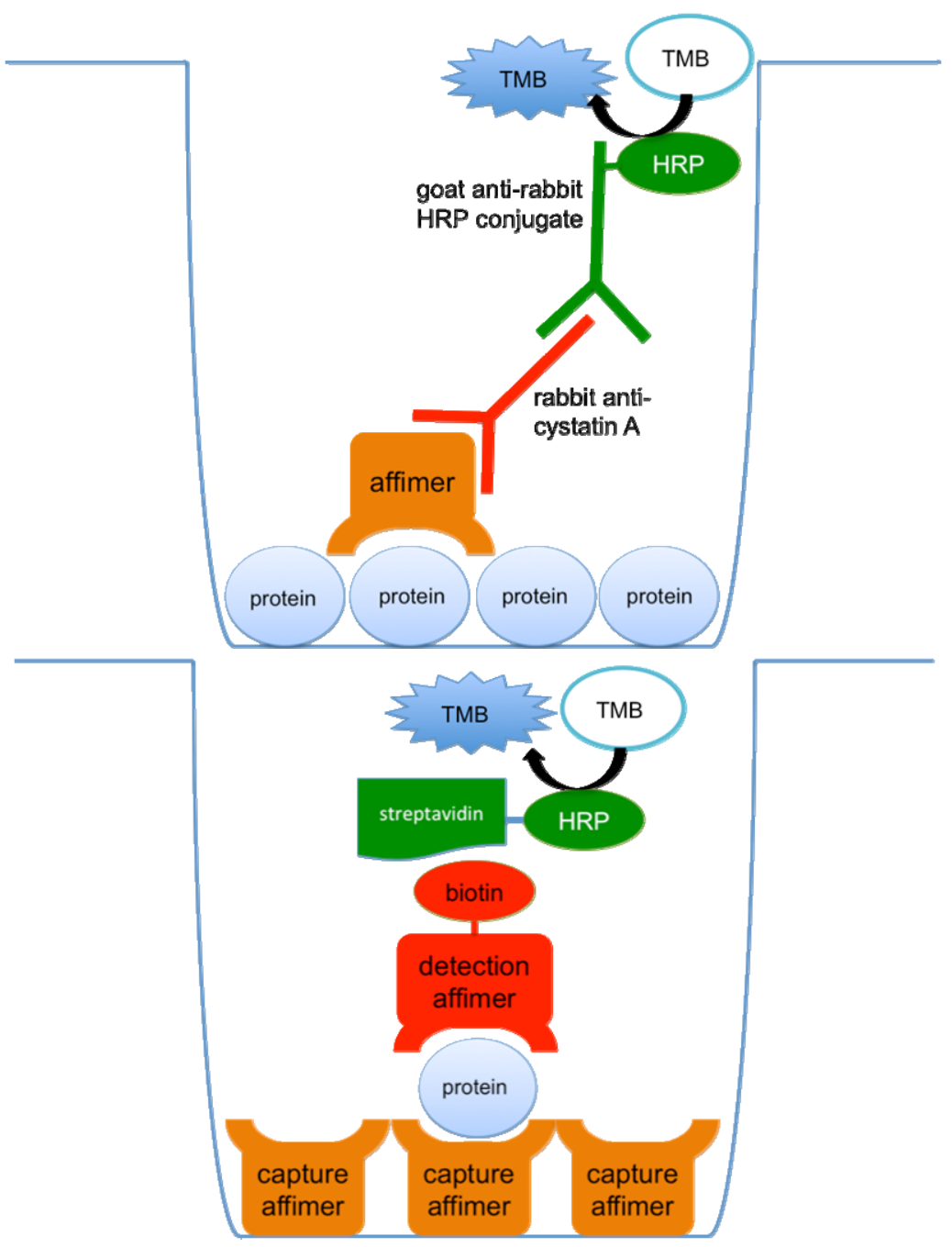

Figure 1. Diagram of ELISA protocols. Indirect ELISA (above): protein (recombinant osteonectin or PEDF) was used to coat the well. Appropriate affimers bind to the protein. Rabbit anti-cystatin A antibody binds to the bound affimer (anti-cystatin A is an antibody against the SQT scaffold) and goat anti-rabbit HRP conjugate antibody binds to anti-cystatin A. HRP catalyses the conversion of TMB substrate to a coloured product, the concentration of which was measured as OD450 following arresting reaction with $\mathrm{HCl}$. Sandwich ELISA (below): the well was coated with capture affimer. Protein is bound to the capture affimer, and a biotinylated detection affimer binds to the same protein at a seperate binding site. Streptavidin HRP conjugate binds to biotin, and HRP catalyses the conversion of TMB substrate to a coloured product, the concentration of which was measured as OD450 following arresting of reaction with $\mathrm{HCl}$.

ticeable colour change or $20 \mathrm{~min}$. OD450 was measured by Multiskan EX plate reader and Ascent software.

This successful protocol was subsequently used to validate naïve affimer "hits" against the PEDF protein (Table 1).

\subsection{Reverse Indirect Affimer ELISA}

An alternative indirect ELISA was carried out to determine whether with the established experimental conditions the proteins osteonectin and PEDF could be de- tected with wells coated with the appropriate affimers. Briefly, a high binding 96-well plate was incubated with a consistent concentration $\left(0.2 \mathrm{mg} \cdot \mathrm{ml}^{-1}\right)$ of the affimers P9I2 and P17F5 (against osteonectin) and P14J4 and P3E1 (against PEDF) overnight at $4^{\circ} \mathrm{C}$.

P9I2 and P17F5 were chosen as the two affimesr which bound to recombinant osteonectin in indirect ELISA (Figure 2), whilst P14J4 and P3E1 were chosen as affimers with highest affinity for recombinant PEDF in indirect ELISA (Figure 3). 


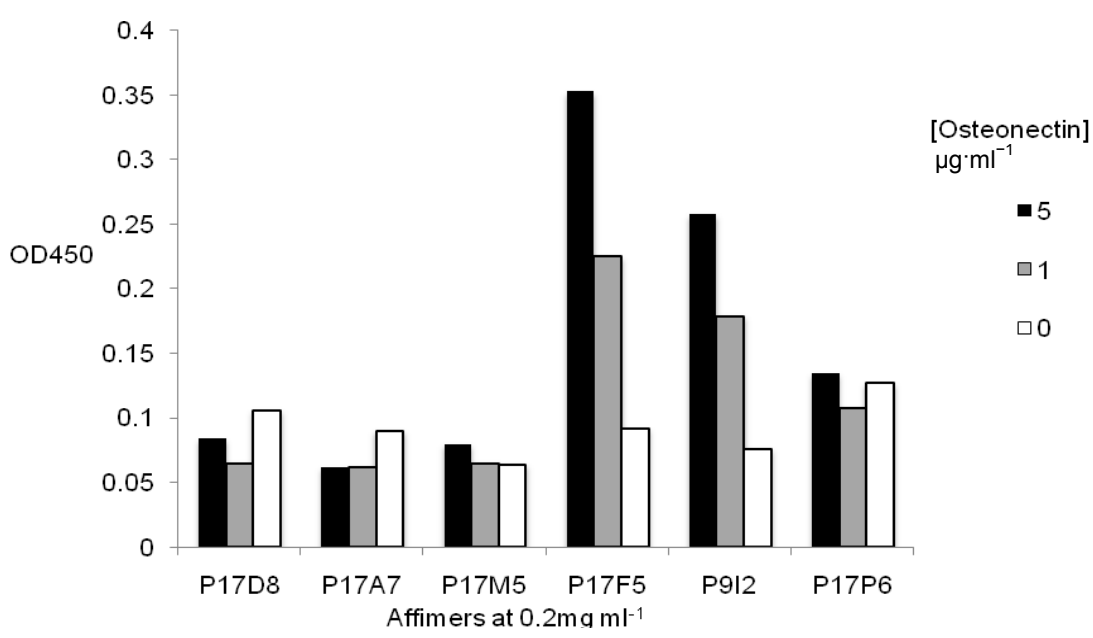

\begin{tabular}{cccc}
\hline & Osteonectin $5 \mu \mathrm{g} \cdot \mathrm{ml}^{-1}$ & Osteonectin $1 \mu \mathrm{g} \cdot \mathrm{ml}^{-1}$ & Osteonectin $0 \mu \mathrm{g} \cdot \mathrm{ml}^{-1}$ \\
\hline P17D8 & 0.084 & 0.065 & 0.106 \\
P17A7 & 0.062 & 0.062 & 0.090 \\
P17M5 & 0.080 & 0.065 & 0.064 \\
P17F5 & 0.353 & 0.225 & 0.092 \\
P912 & 0.258 & 0.179 & 0.076 \\
P17P6 & 0.135 & 0.108 & 0.127 \\
\hline
\end{tabular}

Figure 2. Bar graph to show OD450 measured after incubating osteonectin with affimers identified by affimer microarray in indirect ELISA format. Optical density is proportional to the extent of binding and therefore the affinity of affimers for osteonectin.

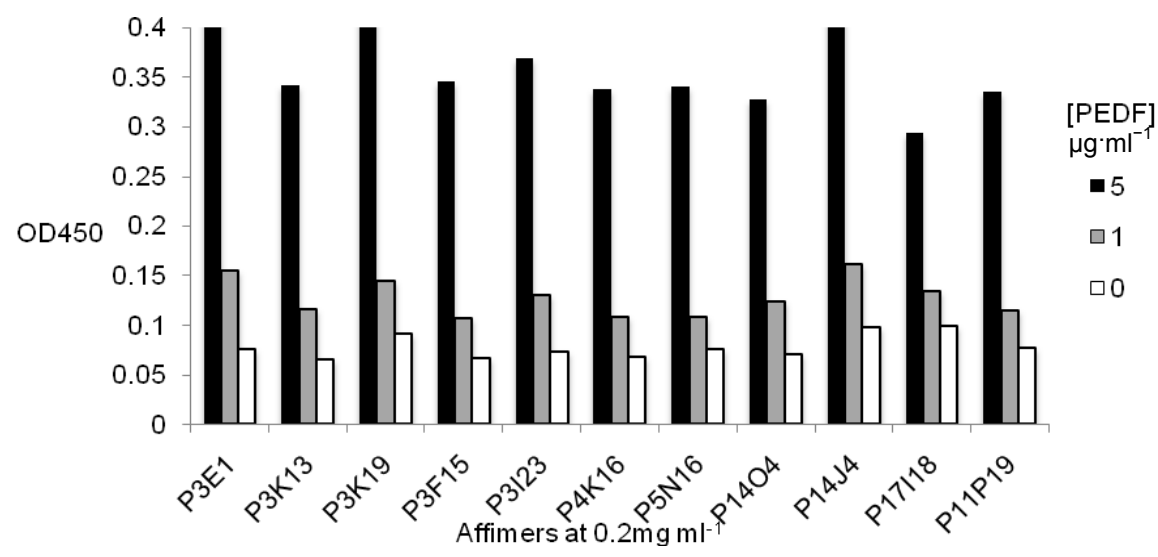

\begin{tabular}{cccc}
\hline & PEDF $5 \mu \mathrm{g} \cdot \mathrm{ml}^{-1}$ & PEDF $1 \mu \mathrm{g} \cdot \mathrm{ml}^{-1}$ & PEDF $0 \mu \mathrm{g} \cdot \mathrm{ml}{ }^{-1}$ \\
\hline P3E1 & 0.496 & 0.155 & 0.075 \\
P3K13 & 0.342 & 0.116 & 0.065 \\
P3K19 & 0.422 & 0.144 & 0.091 \\
P3F15 & 0.345 & 0.106 & 0.066 \\
P3123 & 0.369 & 0.130 & 0.073 \\
P4K16 & 0.338 & 0.108 & 0.068 \\
P5N16 & 0.341 & 0.108 & 0.075 \\
P14O4 & 0.328 & 0.124 & 0.070 \\
P14J4 & 0.418 & 0.161 & 0.097 \\
P17118 & 0.294 & 0.134 & 0.099 \\
P11P19 & 0.335 & 0.115 & 0.077 \\
\hline
\end{tabular}

Figure 3. Bar graph to show OD450 measured after incubating PEDF with affimers identified by affimer microarray in indirect ELISA format. Optical density is proportional to the extent of binding and therefore the affinity of affimers for PEDF.

Affimers were discarded and blocking followed by washing was performed as described previously (see 
indirect affimer ELISA). Recombinant osteonectin and PEDF proteins were applied to the appropriate affimers at concentrations of 5,1 , and $0 \mu \mathrm{g} \cdot \mathrm{ml}^{-1}$ in duplicate and incubation for $1 \mathrm{~h}$ at room temperature on ELISA plate mixer at $250 \mathrm{rpm}$. Washing and then incubation with rabbit anti-osteonectin(Merck) and mouse anti-PEDF (Merck) at a concentration of 1 in 1000 in $3 \%$ BSA in PBS. Washing and then incubation with goat anti-rabbit HRP conjugate (Santa Cruz Biotechnology) and sheep antimouse HRP conjugate (Sigma) as appropriate. Following washing, detection was performed as described previously (see indirect affimer ELISA).

\subsection{Sandwich ELSSA Recombinant Osteonectin and PEDF Validation}

Using the reaction conditions established by the indirect affimer ELISA, a sandwich ELISA was performed to validate the binding of the affimers P17F5 and P9I2 to recombinant osteonectin, and P14J4 and P3E1 to recombinant PEDF in indirect ELISA.

A high binding 96-well plate was coated with each capturing affimer at a consistent concentration $(0.2$ $\mathrm{mg} \cdot \mathrm{ml}^{-1}$ ) overnight at $4^{\circ} \mathrm{C}$. This was followed by blocking and then washing as described previously (see indirect affimer ELISA) and then application of peptide at varying concentrations $\left(5,1\right.$ and $\left.0 \mu \mathrm{g} \cdot \mathrm{ml}^{-1}\right)$ in duplicate with incubation for $1 \mathrm{~h}$ at room temperature with mixing at $250 \mathrm{rpm}$ on ELISA plate mixer. Following washing, 50 $\mu \mathrm{l}$ of a second, biotinylated detection affimer was added at a concentration of $0.2 \mathrm{mg} \cdot \mathrm{ml}^{-1}$, i.e. the affimer P9I2 was used to coat the well and capture osteonectin protein and the affimer P17F5 was used for the detection or binding; and vice versa. The affimers P14J4 and P3E1 were used for the protein recombinant PEDF. The detection affimer was biotinylated to allow detection by application by streptavidin-HRP conjugate.

Briefly, a $0.2 \mathrm{mg} \cdot \mathrm{ml}^{-1}$ affimer solution was buffer exchanged to PBS in order to remove imidazole which would reduced the extent of amine coupled biotinylation. $500 \mu \mathrm{l}$ PBS was applied to Bio-Spin 6 chromatography columns (Bio Rad) followed by centrifugation at $1000 \mathrm{~g}$ for $1 \mathrm{~min}$ four times. Following this, $400 \mu \mathrm{laf}-$ fimer solution was applied followed by centrifugation for $4 \mathrm{~min}$ at $1000 \mathrm{~g}$ as per the manufacturer's instructions [20]. $6.4 \mu \mathrm{l}$ of $0.2 \mathrm{mg} \cdot \mathrm{ml}^{-1}$ EZ-link NHS-SS biotin (Pierce) in DMSO was added to affimer solution and the procedure continued as described previously (see probing of naïve affimer microarray with candidate biomarker peptides).

Following incubation with biotinylated affimer, washing was performed followed by incubation with streptavidin-HRP conjugate (BD Biosciences) 1 in $10003 \%$
BSA in PBS. Following washing, TMB and $\mathrm{HCl}$ were applied as above and OD450 measured.

\section{RESULTS}

\subsection{Probing of Naïve Affimer Microarray}

In total 39 binding affimers were identified against eight out of nine candidate biomarker peptides.

Examples of affimer microarray and "hit" from microarray are shown in Figure 4.

A summary of the "hits" identified in affimer microarray is shown in Table 2.

Affimers that bound to osteonectin and PEDF in affimer microarray, prioritised for further investigation are described in Table 1.

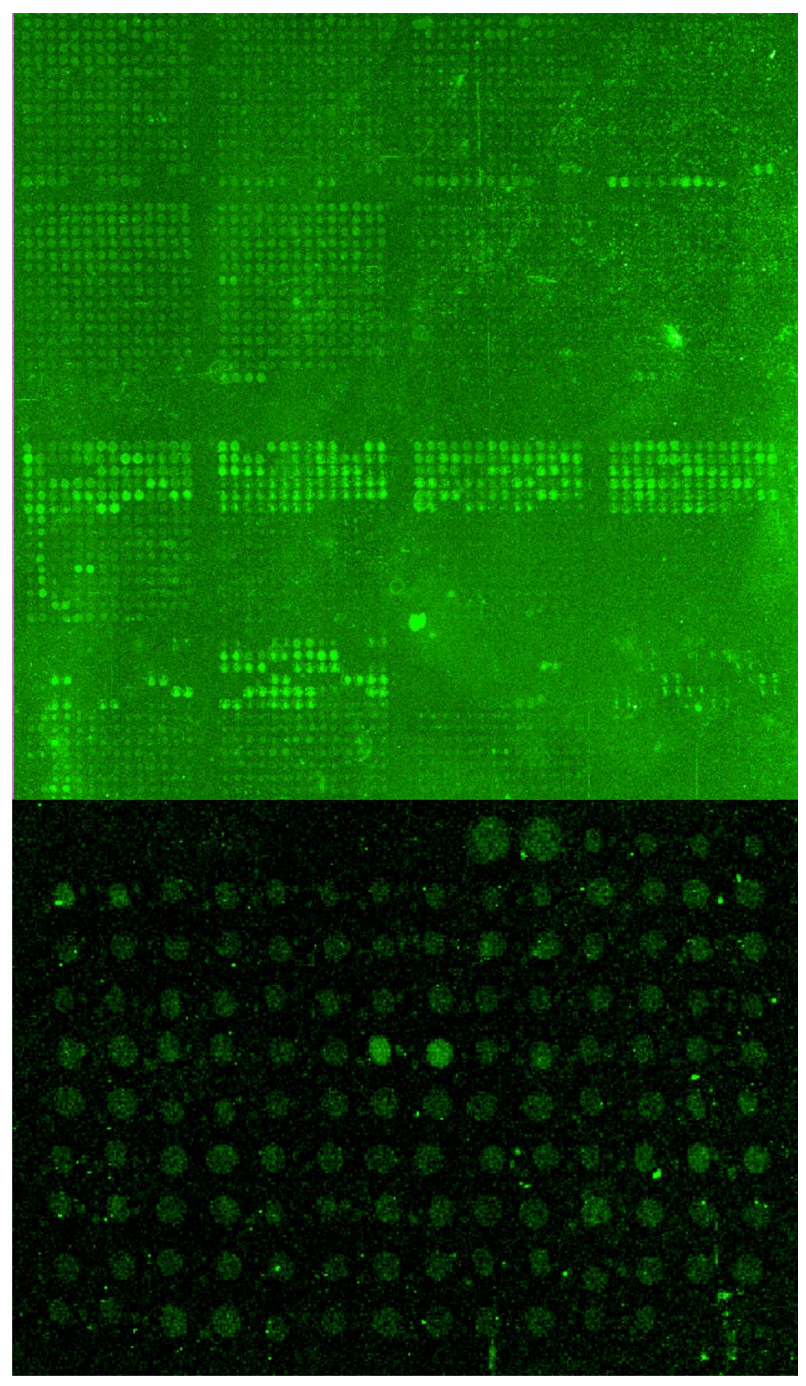

Figure 4. Example microarrays. Showing the appearance of a typical microarray viewed by Genepix 4.0 software (above) and an example of a "hit" (below) (calrecticulin precursor, A. 15. 10. 7,8 .), the two adjacent spots with signal intensity above the background and surrounding spots. 
Table 2. "Hits" obtained by microarray for candidate biomarkers. Each "hit" represents the position of an affimer on microarray. For example: A.1.1.1,2. = Microarray slide A, block 1, row 1, columns 1 and 2 .

\begin{tabular}{|c|c|}
\hline Candidate biomarker & Position of "hit" on microaray \\
\hline \multirow{5}{*}{ Reticulocalbin-3 } & A.4.6.9,10 \\
\hline & A.5.3.9,10 \\
\hline & A. $45.2 .9,10$ \\
\hline & B.21.5.13,14 \\
\hline & B. $31.1 .13,14$ \\
\hline \multirow{3}{*}{$\alpha 2$ chain of type I collagen } & A. $21.10 .11,12$. \\
\hline & A.21. 12. 1,2. \\
\hline & A.6.7.1,2 \\
\hline \multirow{3}{*}{ Reticulocalbin-1 } & A.22.3.1,2 \\
\hline & B.12.1.11,12 \\
\hline & В.37.12.1,2 \\
\hline \multirow{5}{*}{ Tropomyosin 4} & A.3.1.11,12 \\
\hline & A.3.2.9, 10 \\
\hline & A.5.3.9,10 \\
\hline & B.11.9.5,6 \\
\hline & B. $40.14 .7,8$ \\
\hline Calreticulin precursor & $\begin{array}{l}\text { A. } 15 \cdot 10.7,8 \text {. } \\
\text { A. } 28.9 \cdot 7,8 \text {. }\end{array}$ \\
\hline Enolase 1 & No hits generated \\
\hline \multirow{4}{*}{ Actin, alpha 1} & A.3.1.11,12 \\
\hline & A. $30.2 .9,10$ \\
\hline & A. $45.3 .13,14$ \\
\hline & B.17.9.7,8 \\
\hline
\end{tabular}

\subsection{Indirect ELSSA to Validate Osteonectin Binding Affimers}

The affimers P17F5 and P9I2 are able to bind to recombinant human osteonectin in indirect ELISA format. Furthermore, they are able to distinguish between concentrations of 5,1 and $0 \mu \mathrm{g} \cdot \mathrm{ml}^{-1}$. A second ELISA with affimers at $0.02 \mathrm{mg} \cdot \mathrm{ml}^{-1}$ was also carried out but this generated unremarkable results (data not shown). Affimers which were not able to detect osteonectin in ELISA format were hypothesized to be either low affinity binders, or act on binding sites within the structure of the folded recombinant protein (Figure 2).

\subsection{Indirect ELISA to Validate PEDF Binding Affimers}

All affimers were able to distinguish between physiological concentrations of PEDF. A second ELISA with $0.4 \mathrm{mg} \cdot \mathrm{ml}^{-1}$ affimers was completed (data not shown), but as with osteonectin, $0.2 \mathrm{mg} \cdot \mathrm{ml}^{-1}$ was sufficient. As the initial microarray probing was carried out with the recombinant PEDF protein, it was hypothesised that all affimers would be able to detect recombinant PEDF in indirect ELISA, which was the case (Figure 3).

\subsection{Reverse Indirect Affimer ELISA to Validate Osteonectin and PEDF Binding Affimer}

The highest affinity affimers identified against PEDF in indirect ELISA were able to identify PEDF in reverse indirect affimer ELISA (Figure 5). As with previous experiments, affimers were unable to detect the presence of osteonectin in reverse affimer ELISA (data not shown). It is hypothesized that this was due to the nature of the osteonectin antibody, which may have bound to the well despite a thorough blocking protocol.

\subsection{Sandwich ELISA to Validate Osteonectin and PEDF Binding Affimers}

The affimers P14J4 and P3E1 were able to detect recombinant PEDF in sandwich ELISA format, whilst using P14J4 as capturing affimer, P3E1 as detection affimer and vice versa (Figure 6).

\section{DISCUSSION}

Stratified medicine is an approach, which put simply, treats different patients differently. It forms part of a continuum of treatment approaches ranging from personalised medicine at one extreme to the norm of empirical medicine at the other [21]. Patients who despite having the same clinical diagnosis may require different treatments, which are stratified based upon a historically observed difference in response associated with a biomarker signature [21,22]. Hence, the development of stratified medicine requires a shift from a one biomarker per disease approach to one which utilises the molecular signatures of disease in a multiplexed assay [13]. Furthermore, to be widely adopted, such multiplexed biomarker

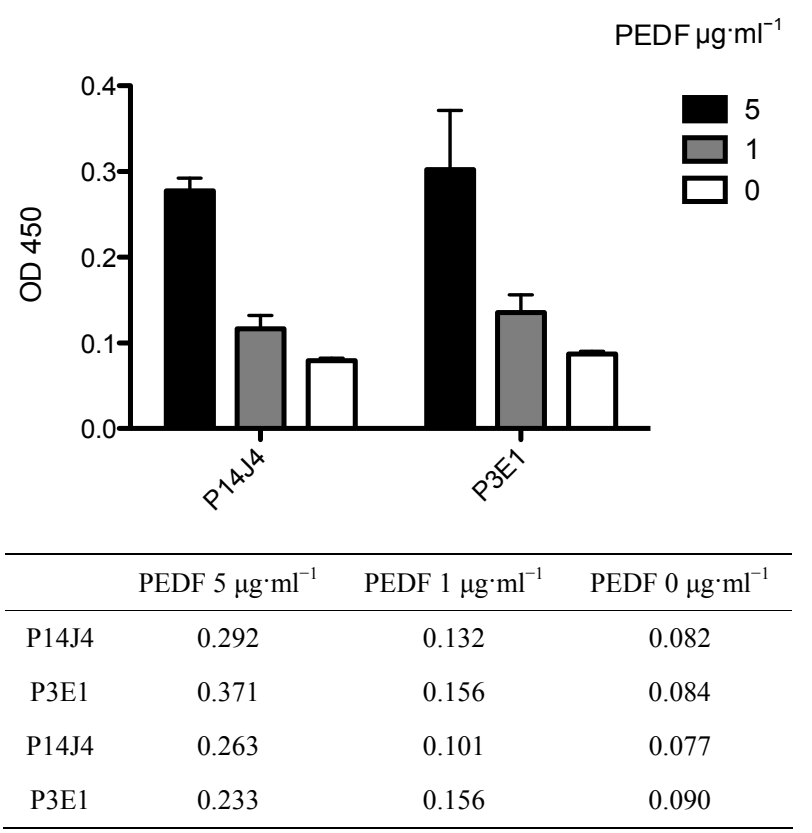

Figure 5. Bar graph to show mean and standard error OD450 measured after incubating affimers identified by affimer microarray with PEDF in reverse indirect ELISA format. Optical density is proportional to the extent of binding and therefore the affinity of affimers for PEDF. 


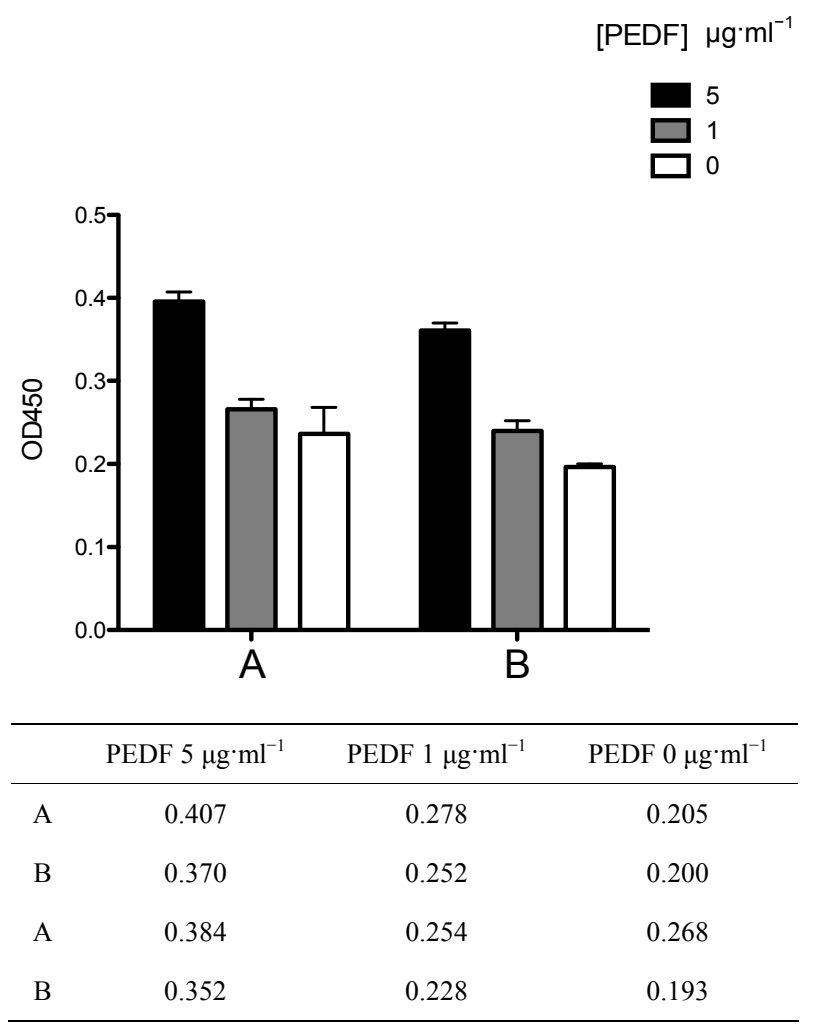

Figure 6. Bar graph to show PEDF and affimers in sandwich ELISA. Data series A represents mean and standard error of OD450 measured for sandwich ELISA protocol which used P14J4 as the capture affimer and P3E1 as the detection affimer. Data series B shows mean and standard error of OD450 measured for sandwich ELISA protocol which used P3E1 as the capture affimer and P14J4 as the detection affimer. Affimers were used against the protein recombinant PEDF.

assays will need to be feasible, reliable, robust, and ideally, rapid [7,13]. SSc is a highly heterogeneous condition in terms of the extent of internal organ involvement and prognosis and therefore it is an ideal candidate for a stratified approach to treatment [2,7].

$\mathrm{SSc}$ is an orphan in respect to disease activity biomarkers and effective treatments, whilst the current gold standard prognostic test, the modified Rodnan skin score has been shown to be inaccurate between observers $[23,24]$. Thus, it is generally accepted that the development of quantitative measurements of disease progression would improve the performance of clinical trials (which most often fail to detect clinically and statistically significant changes in disease activity $[25,26])$ and allow better patient management $[3,9]$. This project represents a step towards this goal for SSc, allowing the implementation of a tool for quantification in the sera of proteins that may reflect disease activity [13].

The biggest challenge in identifying disease biomarkers is not the discovery but in the validation process. Systemic sclerosis has a prevalence of only approxi- mately $1: 10,000$ [27] which is reflected by a relative paucity of clinical samples available for study. The 1500 serum samples held by the European scleroderma research and trials consortium are divided amongst European research groups and priority given to validated platforms (typically $50 \mu \mathrm{l}$ per serum sample). Thus for the validation of several serum biomarkers, a multiplexed assay is extremely advantageous.

To date, the development of multiplexed testing has not heen taken place, most probably due to the nature of antibody molecules (see introduction) and the relatively slow development of novel antibodies [13]. Here, we draw together the work of two very different research groups with the intention of developing a novel, nonantibody technology for the application of identifying biomarkers in systemic sclerosis. We show that by the use of affimer microarray and affimer ELISA, we are able to identify approximately physiological concentrations of soluble proteins in vitro: the mean serum concentration of osteonectin and PEDF in the healthy population has been shown to be $1.62 \pm 0.36 \mu \mathrm{g} \cdot \mathrm{ml}^{-1}$ [28] and $14.6 \pm 3.2 \mu \mathrm{g} \cdot \mathrm{ml}^{-1}$ respectively [29].

The approach of identifying biomarkers obtained from cultured explanted dermal fibroblasts relies upon the assumption that preferentially expressed proteins would also be present in vivo in patient serum. To address this criticism Del Galdo et al. have demonstrated the presence of at least one of these proteins (reticulocalbin-1) in patient serum [3]. However, there remains the possibility that the concentration of this protein and others in serum may either be undetectable or not reflect disease activity.

Another problem associated with the Del Galdo et al. study is that it was conducted with a small patient sample SSc $n=3$, NSF $n=3$ and control $n=3$ [3]. Thus differential changes in protein expression within this small patient sample may not be reflected in a larger cohort of patients. An answer to this criticism is that Del Galdo et $a l$. used an unbiased non-hypothesis driven approach, but managed to identify proteins known to form a part of the fibrotic process ( $\alpha 2$ chain of type I collagen, $\alpha$-smooth muscle actin and osteonectin) suggesting that the findings are a genuine reflection of the profibrotic phenotype [3].

There were several weaknesses with the study design adopted here. The first is that we have developed low affinity binding affimers which work at concentrations of around $0.2 \mathrm{mg} \cdot \mathrm{ml}^{-1}$ but have been shown not to work at concentrations of $0.02 \mathrm{mg} \cdot \mathrm{ml}^{-1}$ (see results). For comparison, the commercially available antibodies utilised during this experiment were used at concentrations of between $0.2-1 \mu \mathrm{g} \cdot \mathrm{ml}^{-1}$. This criticism is addressed by the fact that affimers may be produced in considerably higher quantities, and considerably less expensive than their antibody counterparts. Secondly, higher affinity 
affimers may be engineered relatively simple from the low affinity affimers identified in this experiment (see the final paragraph).

The identification of only low affinity affimers was due to the nature of the affimer microarray. This high throughput screening experiment was carried out with a microarray of $10^{4}(17,000)$ peptide aptamers (affimers). An alternative technology - phage display allows libraryies of complexity of $10^{12}-10^{15}$ peptide aptamers to be developed which allow the screening of many more potential peptide aptamers, and thus there is a higher chance of identifying a high affinity aptamers [16,30]. However, the advantages of peptide aptamer microarrays are that large volumes of data may be generated in a short amount of time (due to automisation) and with relatively small volume samples [31]. Furthermore, this experiment and others like it $[13,19]$ pave the way for the future development of microarrays with much greater complexity.

Another weakness of this study is that an adequate control was not possible for affimer microarray. The control study used was to detect affimers, which were bound to streptavidin, a reagent used in the microarray procedure (see materials and methods). However, there is the possibility that certain affimers are bound to binding sites on the proteins in question, which are shared with other proteins not investigated. However, this criticism becomes less relevant as several validated affimers allow the detection of proteins at multiple binding sites, which are less likely to be shared by unrelated proteins.

A further criticism which may be made against this study is that it failed to validate measurements of relevant proteins in patient sera. Thus the data presented here may be regarded as preliminary.

Since we have demonstrated that the approach of developing a sandwich assay from a high throughput microarray screening is feasible and potentially very informative, we envisage that future work should be done for the complete validation (by ELISA) of all affimers that have been identified by microarray (see Table 2). Furthermore, given the known genetic sequence of the affimer plasmid, it is a relatively simple task to perform studies of site directed mutagenesis and identify affimers with higher affinity for the protein of interest. The characterisation of high affinity affimers would allow a swift implementation at clinical level of a multiplexed affimer based on assay to measure biomarkers of fibrotic activity in SSc sera.

\section{REFERENCES}

[1] Moon, K.W., et al. (2011) The correlation between durometer score and modified Rodnan skin score in systemic sclerosis. Rheumatology International, 32, 24652470 .
[2] Castro, S.V. and Jimenez, S.A. (2010) Biomarkers in systemic sclerosis. Biomarkers in Medicine, 4, 133-147. doi: 10.2217/bmm.09.79

[3] Del Galdo, F., Shaw, M.A. and Jimenez, S.A. (2010) Proteomic analysis identification of a pattern of shared alterations in the secretome of dermal fibroblasts from systemic sclerosis and nephrogenic systemic fibrosis. American Journal of Pathology, 177, 1638-1646. doi:10.2353/ajpath.2010.091095

[4] Furst, D., et al. (2007) Systemic sclerosis-Continuing progress in developing clinical measures of response. Journal of Rheumatology, 34, 1194-200.

[5] Matucci-Cerinic, M., et al. (2009) The complexity of managing systemic sclerosis: screening and diagnosis. Rheumatology (Oxford), 48, 8-13.

doi:10.1093/rheumatology/ken482

[6] Grobner, T. and Prischl, F.C. (2007) Gadolinium and nephrogenic systemic fibrosis. Kidney International, $\mathbf{7 2}$, 260-264. doi:10.1038/sj.ki.5002338

[7] Abignano, G., et al. (2011) Biomarkers in the management of scleroderma: An update. Current Rheumatology Reports, 13, 4-12. doi:10.1007/s11926-010-0140-Z

[8] Moinzadeh, P., et al. (2012) Biomarkers for skin involvement and fibrotic activity in scleroderma. Journal of the European Academy of Dermatology and Venereology, 26, 267-276. doi:10.1111/j.1468-3083.2011.04206.x

[9] S, S. (2012) Stratified medicine approach for systemic sclerosis: State of the art and the need for clinical biomarkers. Unpublished Literature Review for Clinical Sciences Programme.

[10] Clements, P.J., et al. (1993) Skin thickness score in systemic sclerosis: An assessment of interobserver variability in 3 independent studies. Journal of Rheumatology, 20, 1892-1896.

[11] Kaldas, M., et al. (2009) Sensitivity to change of the modified Rodnan skin score in diffuse systemic sclerosis -Assessment of individual body sites in two large randomized controlled trials. Rheumatology (Oxford), 48 , 1143-1146. doi:10.1093/rheumatology/kep202

[12] Crawford, M., Woodman, R. and Ko Ferrigno, P. (2003) Peptide aptamers: Tools for biology and drug discovery. Briefings in Functional Genomics, 2, 72-79. doi:10.1093/bfgp/2.1.72

[13] Song, Q., et al. (2011) Peptide aptamer microarrays: Bridging the bio-detector interface. Faraday Discuss, 149, 79-92, 137-157. doi:10.1039/c005376g

[14] Woodman, R., et al. (2005) Design and validation of a neutral protein scaffold for the presentation of peptide aptamers. Journal of Molecular Biology, 352, 1118-1133. doi:10.1016/j.jmb.2005.08.001

[15] Stadler, L.K., et al. (2011) Structure-function studies of an engineered scaffold protein derived from Stefin A. II: Development and applications of the SQT variant. Protein Engineering Design \& Selection, 24, 751-763. doi:10.1093/protein/gzr019

[16] Murray, E., et al. (2007) Microarray-formatted clinical biomarker assay development using peptide aptamers to anterior gradient-2. Biochemistry, 46, 13742-13751. 
doi:10.1021/bi7008739

[17] Davis, J.J., et al. (2009) Peptide aptamers in label-free protein detection: 2. Chemical optimization and detection of distinct protein isoforms. Analytical Chemistry, 81, 3314-3320. doi:10.1021/ac802513n

[18] (2012) Aptuscan unique affinity reagents. http://www.avacta.com/affinity-reagents

[19] Song, Q., McGonagle, D., et al. (2011) Scannin microbaray identifying protein biomarkers in inflammatory diseases. Leeds Institute of Molecular Medicine.

[20] Nucleic acid purification. http://www.bio-rad.com/webroot/web/pdf/lsr/literature/LI T-507G.pdf

[21] Trusheim, M.R., Berndt, E.R. and Douglas, F.L. (2007) Stratified medicine: Strategic and economic implications of combining drugs and clinical biomarkers. Nature Reviews Drug Discovery, 6, 287-293. doi:10.1038/nrd2251

[22] Hughes, B. (2009) Developing tools for stratified medicine. Nature Reviews Drug Discovery, 8, 919-920. doi:10.1038/nrd3070

[23] Ionescu, R., et al. (2010) Repeated teaching courses of the modified Rodnan skin score in systemic sclerosis. Clinical and Experimental Rheumatology, 28, S37-S41.

[24] Furst, D.E., et al. (1998) The modified Rodnan skin score is an accurate reflection of skin biopsy thickness in systemic sclerosis. Journal of Rheumatology, 25, 84-88.

[25] Postlethwaite, A.E., et al. (2008) A multicenter, randomized, double-blind, placebo-controlled trial of oral type I collagen treatment in patients with diffuse cutaneous sys-

\section{ABBREVIATIONS}

SSc: systemic sclerosis

SQT: stefinA quadruple mutant-Tracy

ELISA: enzyme linked immunosorbant assay

PEDF: pigment epithelium-derived factor

NSF: nephrogenic systemic fibrosis

MRI: magnetic resonance imaging

MRSS: modified Rodnan skin score

Ni-NTA: nickel-nitrilotriacetic acid

BSA: bovine serum albumin

PBS: phosphate buffered saline solution

$[\mathrm{v} / \mathrm{v}]$ : concentration of solution by volume in compareson to diluting solution

PBST: phosphate buffered saline solution with $0.1 \%[\mathrm{v} / \mathrm{v}]$ Tween 20, unless otherwise stated temic sclerosis: I. Oral type I collagen does not improve skin in all patients, but may improve skin in late-phase disease. Arthritis \& Rheumatism, 58, 1810-1822. doi:10.1002/art.23501

[26] Khanna, D., et al. (2009) Recombinant human relaxin in the treatment of systemic sclerosis with diffuse cutaneous involvement: A randomized, double-blind, placebo-controlled trial. Arthritis \& Rheumatism, 60, 1102-1111. doi:10.1002/art.24380

[27] Rull, G. (2011) Systemic sclerosis (scleroderma). http://www.patient.co.uk/doctor/Systemic-Sclerosis-(Scle roderma).htm

[28] Ikuta, Y., et al. (2005) Highly sensitive detection of melanoma at an early stage based on the increased serum secreted protein acidic and rich in cysteine and glypican-3 levels. Clinical Cancer Research, 11, 8079-8088. doi:10.1158/1078-0432.CCR-05-1074

[29] Yamagishi, S., et al. (2006) Elevated serum levels of pigment epithelium-derived factor in the metabolic syndrome. Journal of Clinical Endocrinology \& Metabolism, 91, 2447-2450. doi:10.1210/jc.2005-2654

[30] Davis, J.J., et al. (2007) Peptide aptamers in label-free protein detection: 1. Characterization of the immobilized scaffold. Analytical Chemistry, 79, 1089-1096. doi:10.1021/ac061863z

[31] Laurenson, S., et al. (2011) Development of peptide aptamer microarrays for detection of HPV16 oncoproteins in cell extracts. Analytical Chemistry, 410, 161-170. doi:10.1016/j.ab.2010.10.038

Rpm: revolutions per minute

H: hours

Min: minutes

DMSO: dimethylsulfoxide solution

$\mathrm{G}$ : centrifugal acceleration relative to $\mathrm{g} 9.81 \mathrm{~m} \cdot \mathrm{s}^{-2}$

HRP: horseradish peroxidase

TMB: 3,3',5,5'-tetramethylbenzidine

$\mathrm{HCl}$ : hydrochloric acid

OD: optical density, or absorbance calculated using ELISA plate reader or spectrophotometer. OD450 = optical density at $450 \mathrm{~nm}$, OD600 = optical density at $600 \mathrm{~nm}$.

LB medium: Luria-Beltani medium

$\mathrm{NaCl}$ : sodium chloride

IPTG: isopropyl $\beta$-D-1 thiogalactopyranoside 